\title{
Development of Intelligent Auxiliary System for Customized Physical Fitness and Healthcare
}

\author{
Chung-Chi Huang ${ }^{1, a}$, Hsiao-Man Liư ${ }^{2}$ and Yen-Ting Ke ${ }^{3}$ \\ ${ }^{1}$ Department of Automation and Control Engineering, Far East University, Tainan City, 744, Taiwan, ROC \\ ${ }^{2}$ Department of Leisure and Sports Management, Far East University, Tainan City, 744, Taiwan, ROC \\ ${ }^{2}$ Department of Mechanical Engineering, Far East University, Tainan City, 744, Taiwan, ROC
}

\begin{abstract}
With the advent of global high-tech industry and commerce era, the sedentary reduces opportunities of physical activity. And physical fitness and health of people is getting worse and worse. At present, the shortage of physical fitness instructors greatly affected the effectiveness of health promotion. Therefore, it is necessary to develop an auxiliary system which can reduce the workload of instructors and enhance physical fitness and health for people. But current general physical fitness and healthcare system is hard to meet individualized needs. The main purpose of this research is to develop an intelligent auxiliary system for customized physical fitness and healthcare. It records all processes of physical fitness and healthcare system by wireless sensors network. The results of intelligent auxiliary systems for customized physical fitness and healthcare will be generated by fuzzy logic Inference. It will improve individualized physical fitness and healthcare. Finally, we will demonstrate the advantages of the intelligent auxiliary system for customized physical fitness and healthcare.
\end{abstract}

\section{Introduction}

With the sedentary reduces opportunities of physical activity, the hypo kinetic disease will lead to the chronic diseases. In recent years, developing of customized physical fitness and healthcare system is more and more flourishing. A PDA-Based healthcare system was proposed (C.C.Yang 2002). And users can make inquiry about the information of the nutritional value, calories of a food, and the calories consumed by exercise. Based on his own personal needs, the individual can design an appropriate diet and exercise with the information. It not only help people to manage personal healthcare no matter in sport, diet or medicine issues, but also help doctors diagnose diseases and enhance the efficiency of medical administration and quality. Considering that the most of the instruments price was too high to popularize at present, a simple design and a fair price instrument focused on fitness testing of sit-up was proposed. (J.C. Su et al. 2009). It combined logic electric cable with an inducted machine that matched with an organization of machinery for sit-up testing and passed through the score indication. The whole process was accomplished by logic electric cable and an inducted machine instead of testing by manual operation that could reduce labour costs, avoid mistakes, also enhanced accuracy. And then a web-based physical fitness system was proposed. (J.C. Su et al. 2008). It focus on simple assessment of personal health risk factor, detection and condition assessment of physical fitness, and information of group exercise prescription...etc. A physical fitness system by using the

\footnotetext{
${ }^{\mathrm{a}}$ Corresponding author: cchuang@cc.feu.edu.tw
}

fuzzy theory is proposed (H.C. Wu 2013). It can increase the learning effects and improve the accuracy of training selection. So that lower training costs can be achieved by using the fuzzy theory to estimate the effective assessment for physical fitness test. An automated interactive exercise coaching system using the Microsoft Kinect was developed (F. Ofli et al. 2015). The system guides users through a series of video exercises, tracks and measures their movements, provides real-time feedback, and records their performance over time. The system consists of exercises to improve flexibility, balance, endurance, and strength, with the aim of improving performance of daily activities and reducing fall risk. A homecare sensory system that assesses the elder's physical fitness through quantifying their home rehabilitation exercises was proposed (C.Y. Chiang et al. 2011). The sensory system used a tri-axial accelerometer to collect the motion acceleration and transmitted the data through wireless personal area networks in the home environment. Through the quantification method for the senior adults, the proposed system attempted to provide appropriate exercise advises for better health administration. To sum up the above, it is very important to develop a customized auxiliary system which can enhance physical fitness and health for people.

\section{Intelligent customized physical fitness and healthcare system}


In this paper, an intelligent assessment and prediction system was proposed to construct an intelligent auxiliary system for complete physical fitness platform system. It constructs a complete physical fitness platform which uses fuzzy logic method to infer customized individual prescriptions according the age, body, fitness and strength. It contains two parts: (1) Assessment for customized physical fitness and healthcare (2) Prediction for customized physical fitness and healthcare. And it uses the architecture of cloud-dust based intelligent system to realize intelligent auxiliary system for customized physical fitness and healthcare. It showed as figure 1.

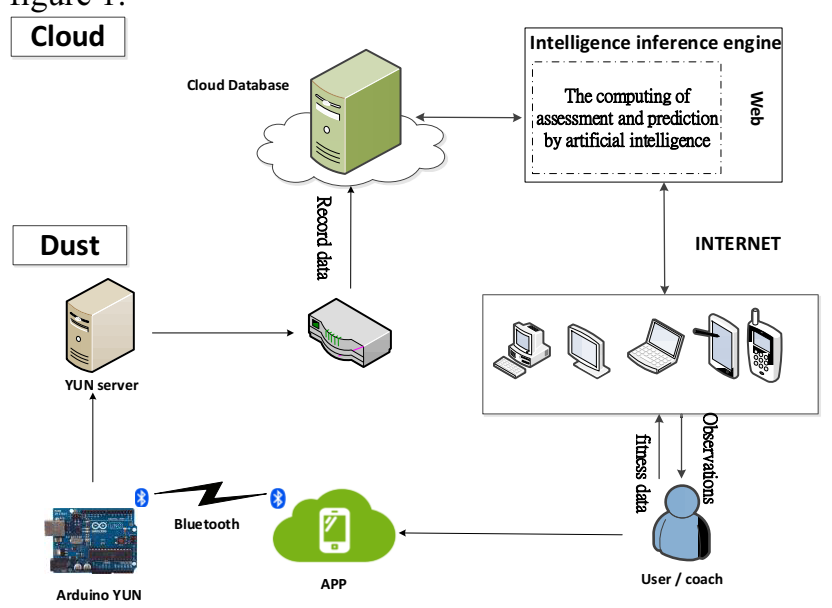

Figure 1. The architecture of cloud-dust based intelligent auxiliary system for customized physical fitness and healthcare

\section{Records and surveillance for customized physical fitness and healthcare}

The App of mobile devices is designed by Zigbee, WiFi, and Bluetooth. The maximum heart rate, the effective heartbeat, the current heart rate and average heart rate will be calculated by using microprocessor to sense data of heartbeats per 5 seconds. It was shown as figure 2 .

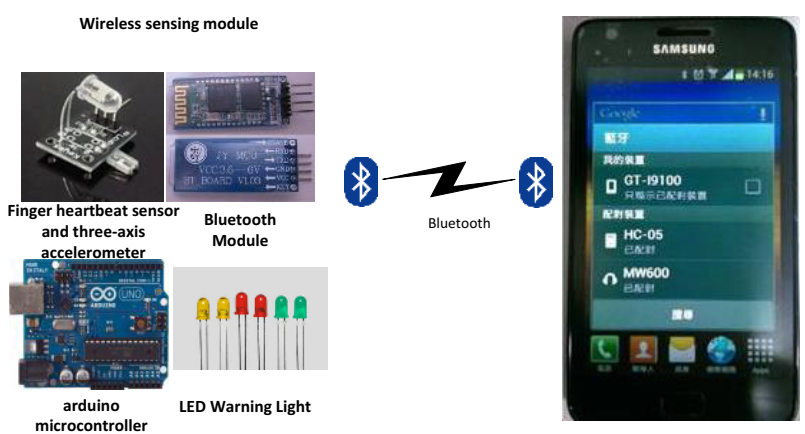

Figure 2. Records and surveillance of heartbeats for customized physical fitness and healthcare

As figure 3 shown, the LED will show the alerts. The green light is showed if the heart rate of the user is in the range of $0-65 \%$. The yellow light is showed in the range of $65-85 \%$ and the red light is showed in the range of $85-$ $100 \%$. It will provide a voice to alert the user and the current state of the coach to keep the sports safety if the red light shows for three times simultaneously. The flowchart of records and surveillance for customized physical fitness and healthcare was shown as figure.

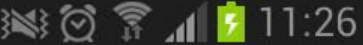
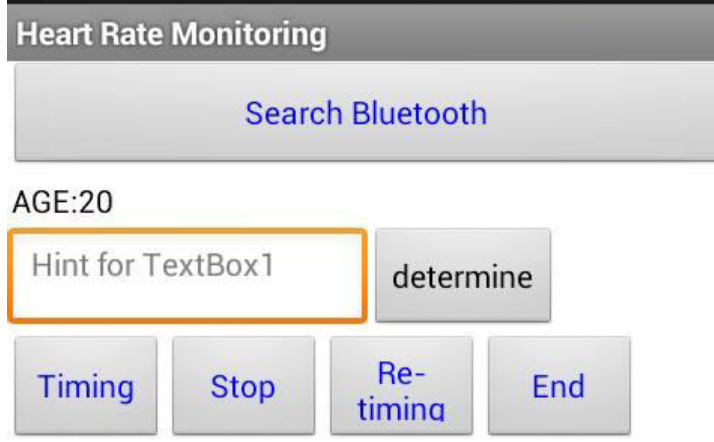

Maximum heart rate : 170

Effective heartbeat interval : 130-170

Currently heartbeat : 97

Average heart rate : 90

$00: 03: 15$

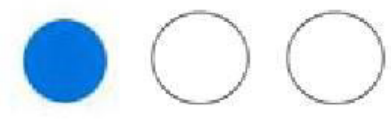

0 65\% 65 85\% 85 100\%

Figure 3. The alert lights of heartbeat states in the system.

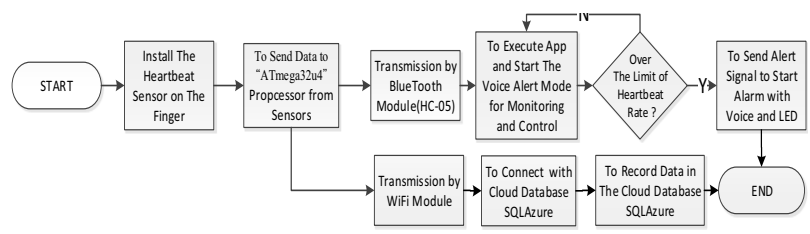

Figure 4. The flowchart of records and surveillance for customized physical fitness and healthcare.

\section{Intelligent assessment for customized physical fitness and healthcare system}

Considering the input parameters as muscular endurance, flexibility and the 3-minute step test, use fuzzy inference to generate the results of assessment for customized physical fitness and healthcare system. The flowchart of assessment using fuzzy inference for customized physical fitness and healthcare system is shown as figure 5 .

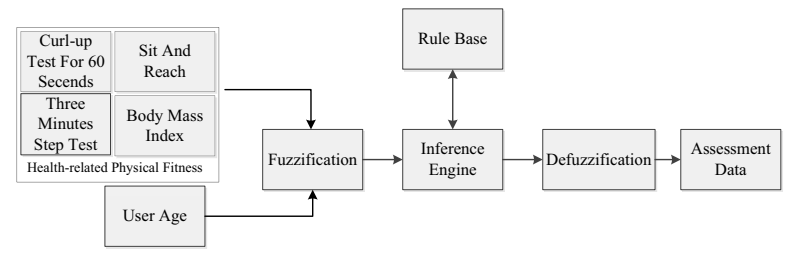

Figure 5. The flowchart of assessment using fuzzy inference for customized physical fitness and healthcare system

The input parameters as muscular endurance, flexibility and the 3-minute step test will be sent to cloud database. 
And then the results of assessment by the computing of cloud fuzzy engine will be generated and showed in webbased human-machine-interface. The architecture of assessment using fuzzy inference for customized physical fitness and healthcare system is shown as figure 6 .

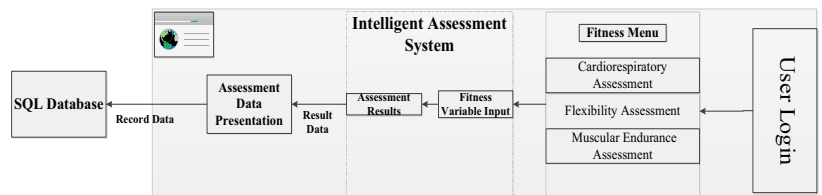

Figure 6. The architecture of assessment using fuzzy inference for customized physical fitness and healthcare system.

The process of fuzzy inference for Health-related Physical Fitness (HRPF) is as the followings:

Step 1. To build up the model of customized physical fitness and healthcare system as figure. 7. And then set Cardiorespiratory-Fitness,

Muscular-Endurance, Flexibility and BMI as input parameters and HRPF as output parameter.

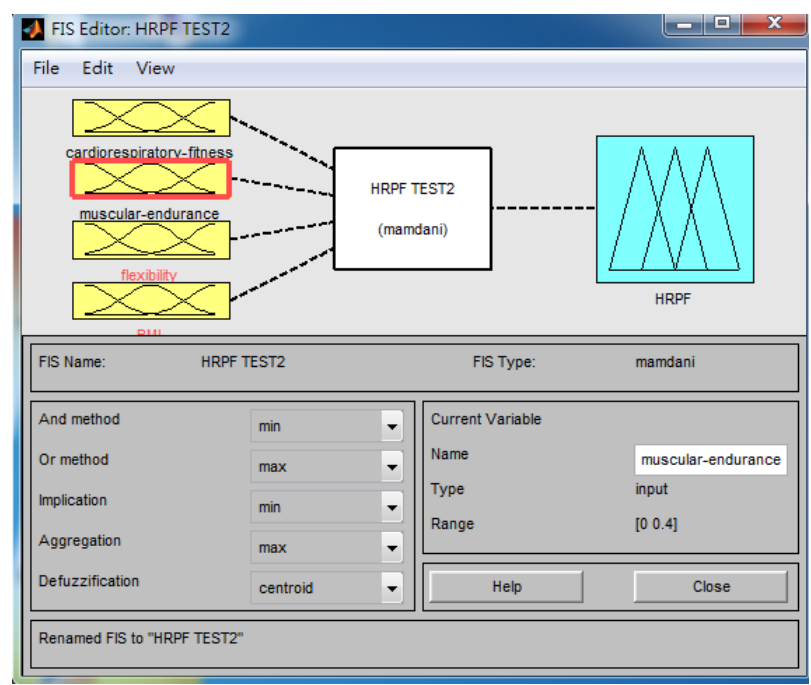

Figure 7. The model of customized physical fitness and healthcare system.

Step 2. To set the parameters of cardiorespiratory-fitness as $0-0.75$ and the membership as $\mathrm{S}(0,0,0.54), \mathrm{M}(0.5$, $0.575,0.65), \mathrm{L}(0.62,0.75,0.75)$. It is showed as figure 8 .

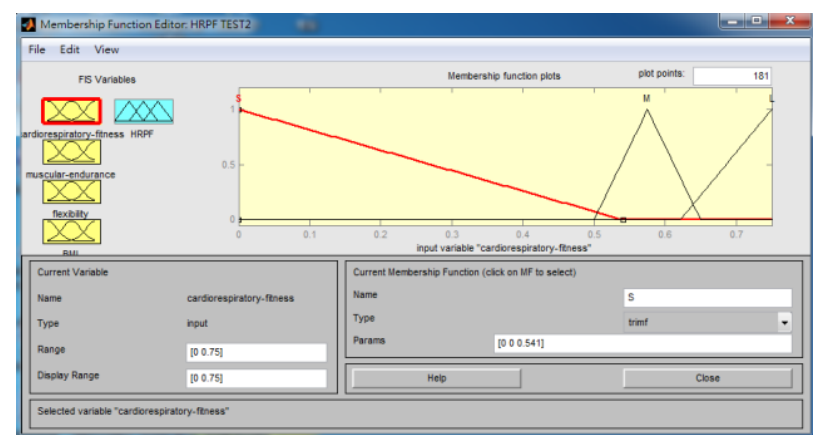

Figure 8. Membership setting for cardiorespiratory fitness.

Step 3. To set the parameters of muscular endurance as 0 0.4 and the membership as $\mathrm{S}(0,0,0.2), \mathrm{M}(0.1,0.2,0.3)$, $\mathrm{L}(0.25,0.4,0.4)$. It is showed as figure 9

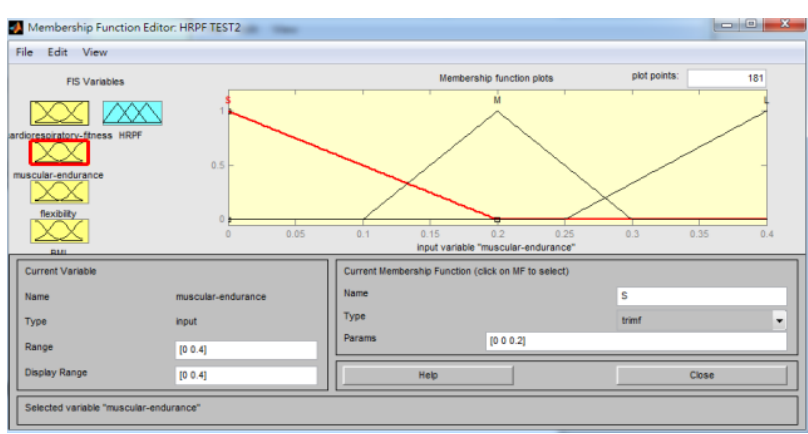

Figure 9. Membership setting for muscular endurance.

Step 4. To set the parameters of flexibility as $0-0.1$ and the membership as $\mathrm{S}(0.01,0.01,0.05), \mathrm{M}(0.02,0.055$, $0.09), \mathrm{L}(0.06,0.1,0.1)$. It is showed as figure 10 .

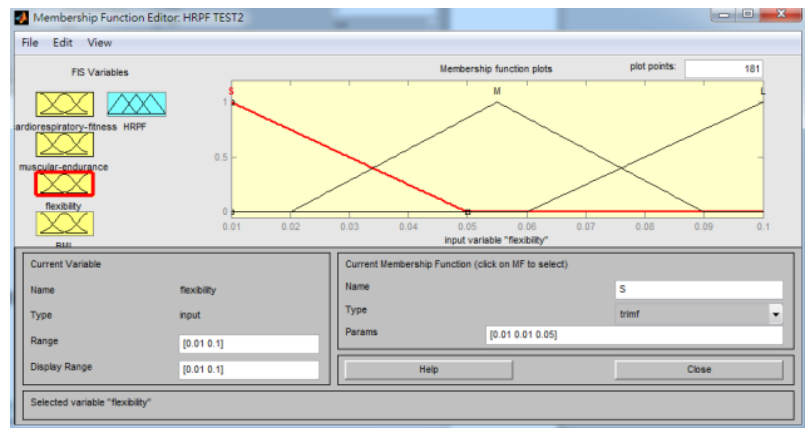

Figure 10. Membership setting for flexibility.

Step 5. To set the parameters of BMI as $0-0.4$ and the membership as $\mathrm{S}(0,0,0.2), \mathrm{M}(0.18,0.24,0.3), \mathrm{L}(0.27$, $0.4,0.4)$. It is showed as figure 11 .

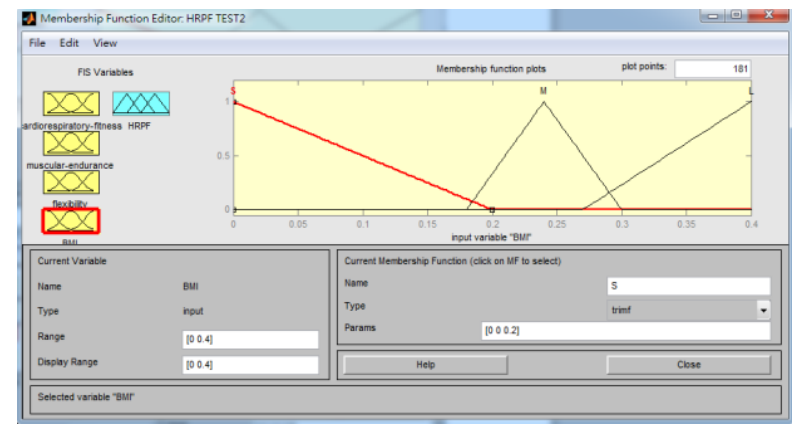

Figure 11. Membership setting for BMI.

Step 6. To set the parameters of HRPF as $0-1$ and the membership as $\mathrm{VS}(0,0,0.25), \mathrm{S}(0,0.25,0.5), \mathrm{M}(0.25$, $0.5,0.75), \mathrm{L}(0.5,0.75,1), \operatorname{ML}(0.5,0.75,1)$. It is showed as figure 12 .

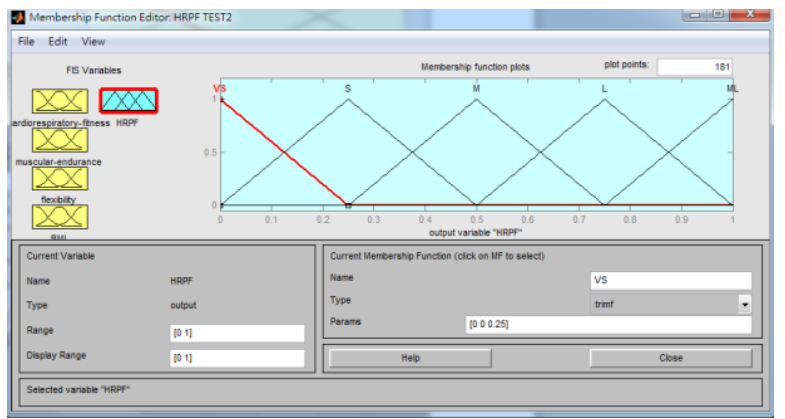

Figure 12. Membership setting for HRPF. 
Step 7. To set 81 rules for HRPF. It is showed in figure 13. Cardiorespiratory fitness is a main item for HRPF.

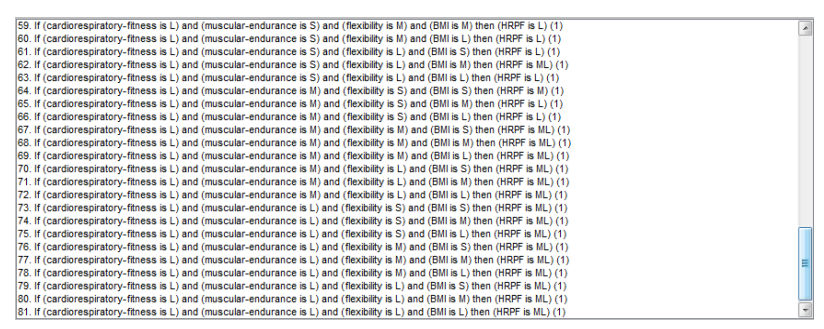

Figure 13. Rules setting for HRPF.

Step 8. The results of fuzzy inference for Health-related Physical Fitness (HRPF) by inputting CardiorespiratoryFitness, Muscular-Endurance, Flexibility and BMI. If Cardiorespiratory-Fitness $=0.405$ (S Area), MuscularEndurance $=0.182$ (M Area), Flexibility $=0.074$ (M Area and $\mathrm{L}$ Area), $\mathrm{BMI}=0.238$ (M Area), the system inferred $\mathrm{HRPF}=0.438$ (M Area). It means the status of user's Physical Fitness is "Middle". It is showed as figure 14.

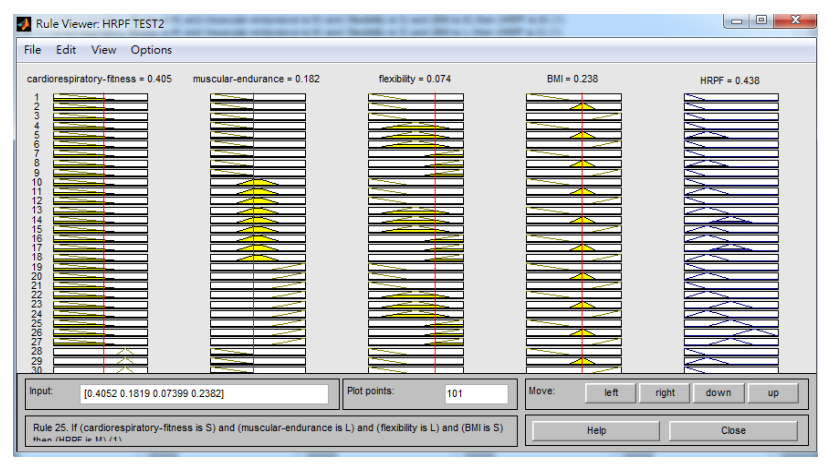

Figure 14. Fuzzy inference for HRPF.

\section{Intelligent prediction for customized physical fitness and healthcare}

Considering the historical records of muscular endurance, flexibility and the 3-minute step test, use neural network to generate the results of trend prediction for customized physical fitness and healthcare system. The flowchart of trend prediction using neural network method for customized physical fitness and healthcare system is shown as figure 15. And the architecture of intelligent inference using neural network method is shown as figure 16 .

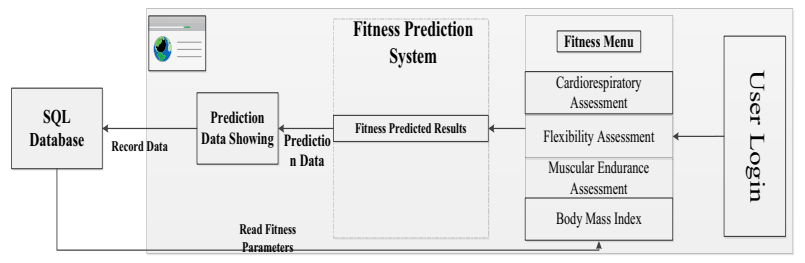

Figure 15. The flowchart of trend prediction using neural network method for customized physical fitness and healthcare.

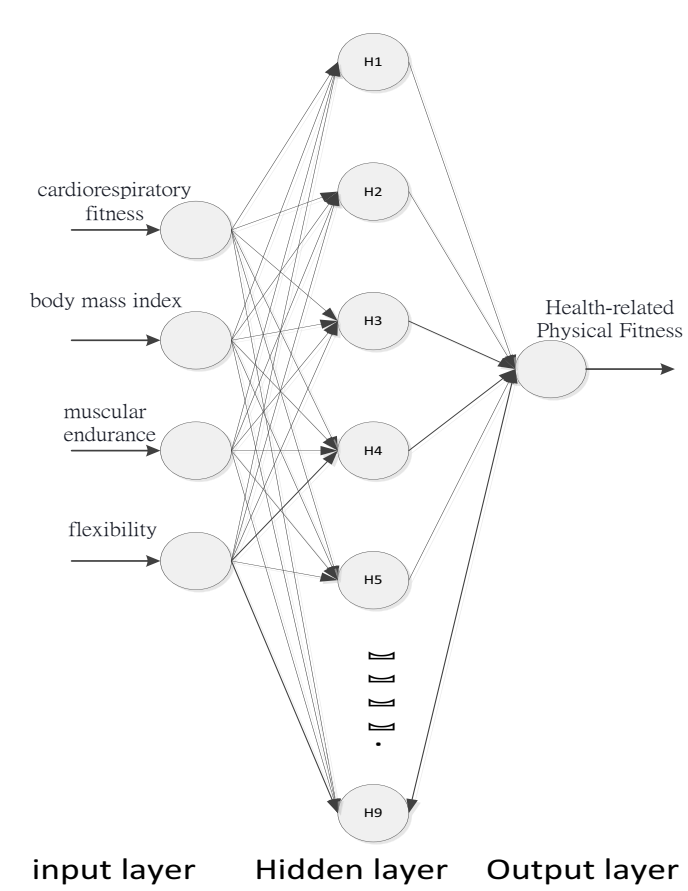

Figure 16. The architecture of intelligent inference using neural network method for trend prediction of HRPF.

Artificial neural network was training by the historical records of muscular endurance, flexibility and the 3minutes step test for trend prediction of HRPF. Based on cloud-dust architecture, the trend prediction of HRPF will be shown in mobile devices or PCs. It is shown as figure 17.

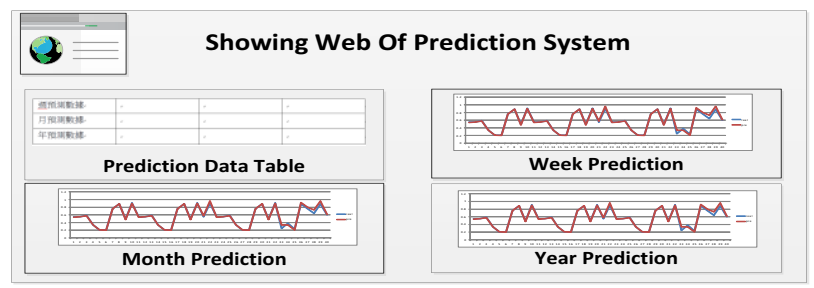

Figure 17. The trend prediction of HRPF based on cloud-dust architecture

\section{Results and discussions}

We can find the performance of intelligent assessment and prediction for customized physical fitness and healthcare system from the experimental results in figure 18 and figure 19. It shows the system has an excellent performance of assessment and trend prediction for customized physical fitness and healthcare system.

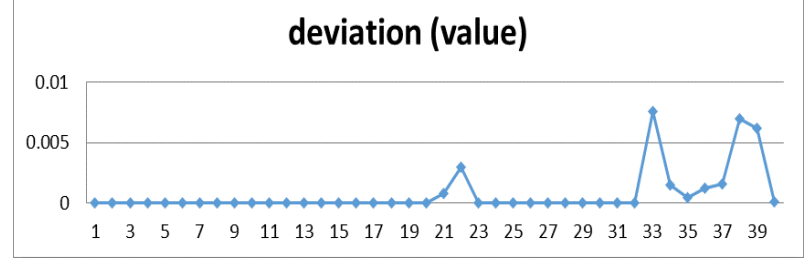

Figure 18. Total deviation of assessment 


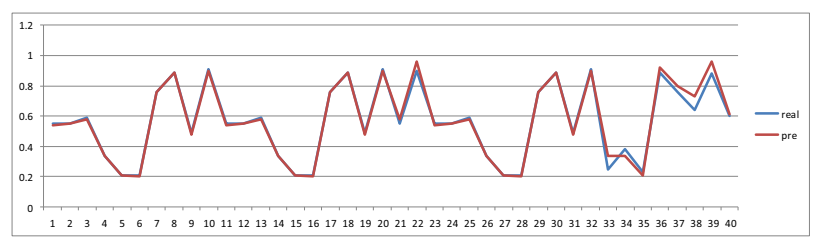

Figure 19. Trend comparison of actual and prediction results

\section{Conclusions}

The results of assessment and prediction for customized physical fitness and healthcare system will be generated by inference of fuzzy logic and neural network. It will improve individualized physical fitness and healthcare. Finally, we will demonstrate the advantages of the intelligent assessment and prediction for customized physical fitness and healthcare system. In order to reduce labour costs, avoid mistakes, also enhanced accuracy, the intelligent system plays an important role in HRPF field. By the experimental results, we can find it meets the both needs of the efficiency and the lowest cost for intelligent assessment and prediction for customized physical fitness and healthcare system.

\section{Acknowledgment}

We would like to thank the Ministry of Science and Technology of the Republic of China (Taiwan) for financial support of this research under contract numbers MOST 104-2622-H-269 -002 -CC3

\section{References}

1. C.C. Yang, The Study of Portable Personal Health Managment System (2002)

2. C.Y. Chiang et al., Quantification of Home Rehabilitation Exercise for the Elder's Physical Fitness Monitoring (2011)

3. F. Ofli et al., Design and Evaluation of an Interactive Exercise Coaching System for Older Adults: Lessons Learned (2015)

4. H.C. Wu, Physical Fintness Fuzzy Assessment, (2013)

5. J.C. Su et al., The Research of the Development on Electric Sit-up Testing System (2009)

6. J.C. Su et al., The Development of Fitness Testing Software System (2008) 